This item was submitted to Loughborough's Research Repository by the author.

Items in Figshare are protected by copyright, with all rights reserved, unless otherwise indicated.

\title{
Reasoning consistently about inconsistency
}

PLEASE CITE THE PUBLISHED VERSION

PUBLISHER

(C) IEEE

VERSION

VoR (Version of Record)

LICENCE

CC BY-NC-ND 4.0

\section{REPOSITORY RECORD}

Hinde, Chris J., Robert S. Patching, R.G. Stone, Daniela Xhemali, and Stephen A. McCoy. 2019. "Reasoning Consistently About Inconsistency". figshare. https://hdl.handle.net/2134/4068. 
This item was submitted to Loughborough's Institutional Repository (https://dspace.lboro.ac.uk/) by the author and is made available under the following Creative Commons Licence conditions.

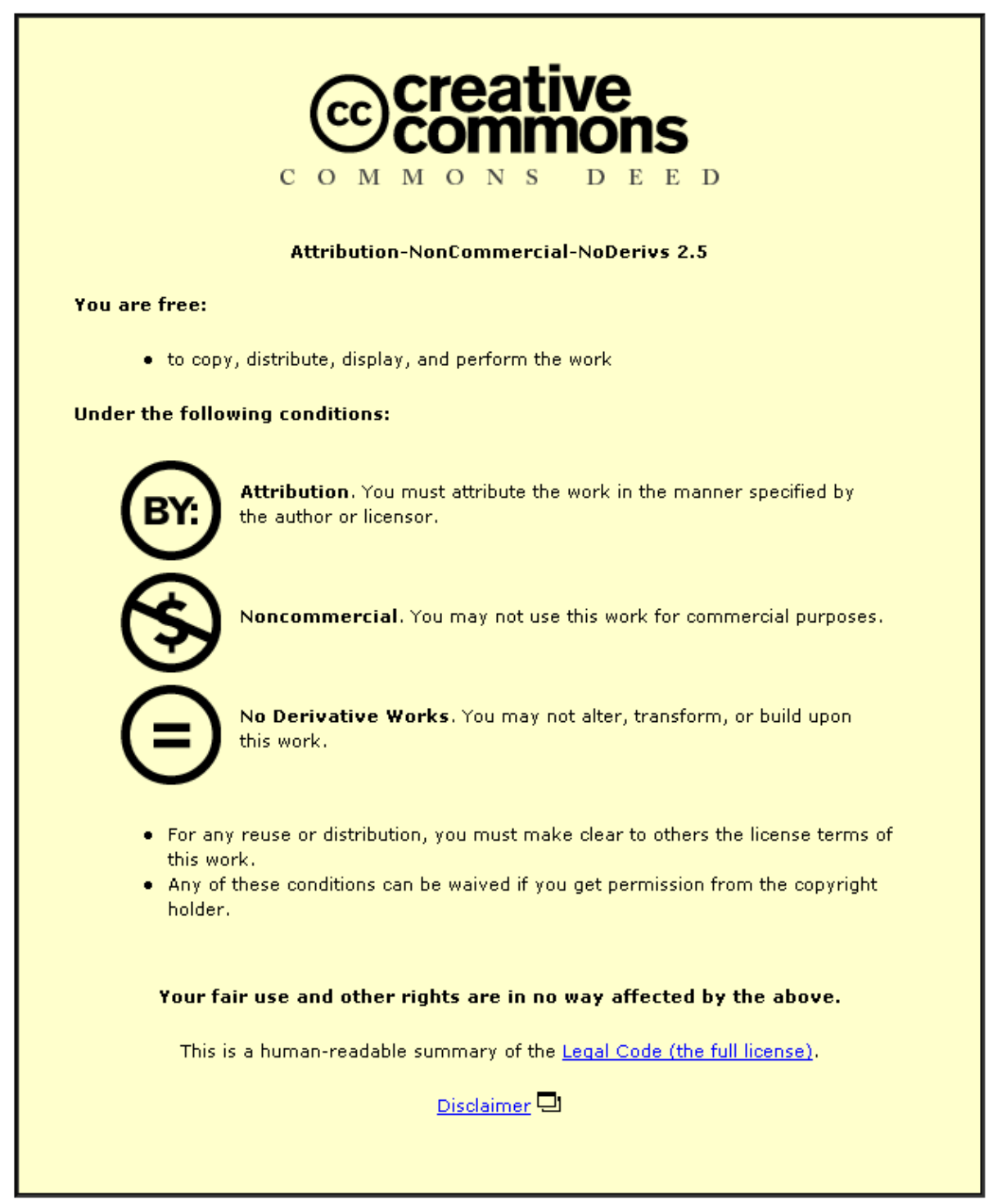

For the full text of this licence, please go to: http://creativecommons.org/licenses/by-nc-nd/2.5/ 


\title{
Reasoning Consistently about Inconsistency
}

\author{
C.J. Hinde, R.S. Patching, R.G. Stone, D. Xhemali and S.A. McCoy
}

\begin{abstract}
Patching et al. and Hinde et al. in their work on truth-space mass assignments, presented a semantic unification function and a semantic separation function for mass assignment logic that dealt with inconsistency. This paper takes these two functions and while preserving the outside inconsistencies shows how inconsistency can be reasoned about in a consistent manner. This means that inconsistency that arises outside the system need not enter the system, but needs to be represented within the system, and can therefore be extracted appropriately as output from the system to emerge as inconsistency on the outside. The internal reasoning system need therefore only concern itself with belief in truth, falsity and uncertainty.
\end{abstract}

\section{Introduction}

In earlier papers two operators were developed to introduce evidence from an arbitrary object space into truth space (1) and to extract object information from truth space (2), Figure 1 shows the relationships. In both cases the inconsistency present in the evidence was preserved to be used within the reasoning system. We are concerned with reasoning about rather than with inconsistency. If a system can be created that allows reasoning about inconsistency without having to introduce inconsistency then the mechanisms of the system are greatly simplified. The operation of semantic unification as introduced by Baldwin (3) is comparable to conditionalization (4) over interval probabilities. Semantic unification is further defined in Section IV-A. Neither work offers a treatment of unification or conditionalization as applied to inconsistent evidence, however Dekhtyar defines consistency. Separation has been referred to as abduction. Abduction is the process which allows the precondition a of Òa entails bó to be inferred from the consequence b. Separation is a similar process whereby we perform the inverse operation of semantic unification to calculate what the effect on the world is of a set of claims that may arise or be present in, a reasoning system. It is not defuzzification which may be defined as the process of producing a quantifiable result from a fuzzy set. Separation induces a fuzzy set in object space from a claim in truth space. In the sense that a fuzzy set in object space can entail a claim in truth space then separation is indeed abduction, Baldwin (3) defines the operation but does not give it a name. Semantic separation seems to be

C.J. Hinde is with the Department of Computer Science, Loughborough University, Loughborough, LE11 3TU, UK (phone: +44 1509 635702; email: c.j.hinde@1boro.ac.uk).

R.S. Patching is with the Department of Computer Science, Loughborough University, Loughborough, LE11 3TU, UK (email: research@woollythinking.co.uk).

D. Xhemali is with the Department of Computer Science, Loughborough University, Loughborough, LE11 3TU, UK (email: d.xhemali@lboro.ac.uk).

R.G. Stone is with the Department of Computer Science, Loughborough University, Loughborough, LE11 3TU, UK (email: R.G.Stone@lboro.ac.uk). S.A. McCoy is with Hazid Technologies Ltd. (email: S.McCoy@hazid.com). a reasonable complementary name to semantic unification. Again, no literature exists apart from Hinde (2) that provides a separation operation that deals with inconsistency.

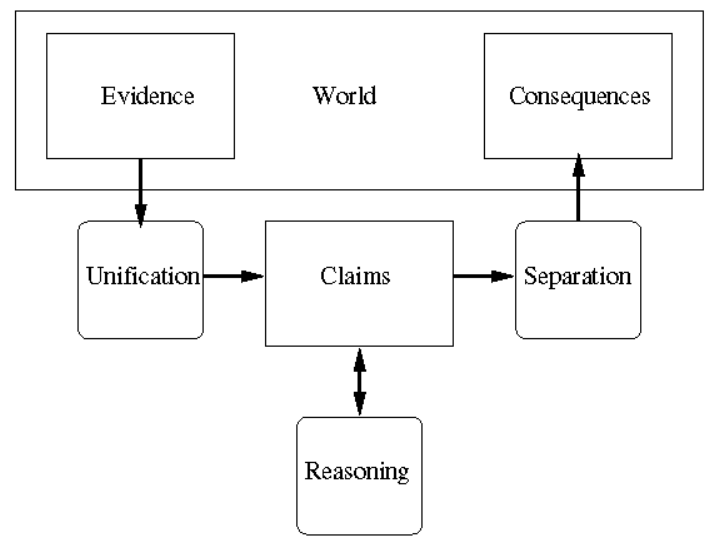

Fig. 1. Showing the flow through unification, reasoning and separation. Evidence is unified into claims, the claims form the basis for the reasoning and then the consequences are separated from the claims to become real world values.

\section{A. Overview}

Semantic unification and separation are treated as a linked pair of functions in order to explore possible combined choices. Section II introduces mass assignment sufficiently to understand the paper, with an introductory section on the notation used. Section III covers other related work which underpins this work, including the concepts of semantic unification and separation. Semantic unification maps knowledge from an arbitrary domain to truth-space $\left(2^{\left\{T^{\top}, \perp\right\}}\right)$ and is explained in Section IV-A. Semantic separation moves knowledge from truth-space $\left(2^{\{\top, \perp\}}\right)$ to an arbitrary domain and is explained in Section IV-C. By considering both operators together we find that there are more choices than before and we can then make decisions about how we wish to reason about things.

\section{Mass Assignment}

Mass assignment (MA) theory was introduced in 1991 by Baldwin (5) and later implemented in a programming language. It is firmly based upon the counting of events and has strong links to probability. Mass is a precise amount of probability assigned to elements of the power set of a domain (i.e. sets of events), rather than individual events themselves. Each MA that has no mass assigned to the empty set describes a family of probabilities. 


\section{A. Notation}

In this paper $X$ and $Y$ represent generic mass assignments and $R$ is the result of a generic semantic unification (see section IV-A). $Y^{\prime}$ is the result of a generic semantic separation (see section IV-C). $\mathbb{C}$ is a generic domain.

$\top$ is verum, a symbol denoting true and only true; $\perp$ is falsum, a symbol denoting false and only false. Together they make the set of Boolean values $\{T, \perp\}$. We choose to use $T$ rather than $t$ because $\{\top\}$ will be derived from $\{\top, \perp\}$ by eliminating $\perp$ whereas $t$ will represent positive evidence for true adding it to $\emptyset$ to give $\{t\}$. Belnap (6) builds evidence from ignorance and so we use $\{t, f\}$ to represent the build up of evidence and $\{\top, \perp\}$ to represent restriction of possibilities. Whereas $\{\top, \perp\}$ allows both possibilities, $\{\top\}$ indicates that true and only true remains, whereas btrue indicates that there is evidence for $t$ but there remains the possibility that there might be evidence for $f$.

\section{B. Mass assignment basics}

The MA $(X)$ is defined on the domain $\mathbb{C}$ and is written as:

$$
\begin{array}{r}
X=X_{1}: x_{1}, \ldots, X_{n}: x_{n} \\
\text { where } \sum_{i=1}^{n} x_{i}=1.0 \text { and } X_{i} \in 2^{\mathbb{C}}
\end{array}
$$

The powerset of the domain $\mathbb{C}$ is denoted $2^{\mathbb{C}} . X_{i}$ is a subset of the domain $\mathbb{C}$ (i.e. $X_{i} \in 2^{\mathbb{C}}$ ) and $x_{i}$ is the amount of mass assigned to that set. Normally a focal element $X_{i}$ is only written if $x_{i}$ is non-zero.

As stated, a MA describes a family of probability distributions and a restriction of a mass assignment corresponds to a subset of that family. MA theory has many types of restriction, which move mass between focal elements. As soon as combination of evidence arises the theory of MA departs from Dempster/Shafer. MA supports the standard fuzzy set operators of intersection and union as it does others. In addition, the truth functions of conjunction, disjunction, negation, etc. exist for MAs defined upon the domain of Boolean values (i.e. $\{\top, \perp\}$ ). MA operators are evaluated by creating a mass assignment tableau, as described in (3).

If a mass assignment is to correspond to a family of probability distributions then $\mathrm{m}(\emptyset)=0$. Mass assignment theory (5) counts $m(\emptyset)$ in its belief measure, the Transferable Belief Model (TBM) (7), on the other hand, does not.

Following (1) the two belief functions are denoted as $\mathrm{Bel}^{+}()$if it includes the empty set and $\mathrm{Bel}^{-}()$if it does not, the two plausibility functions are also defined:

$$
\begin{aligned}
& \mathrm{Bel}_{X}^{+}\left(X_{i}\right)=\sum_{Y_{j} \subseteq X_{i}} \underset{X}{\operatorname{m}}\left(Y_{j}\right) \quad \mathrm{Bel}_{X}^{-}\left(X_{i}\right)=\sum_{\emptyset \neq Y_{j} \subseteq X_{i}} \underset{X}{\operatorname{m}}\left(Y_{j}\right) \\
& \mathrm{Pl}_{X}^{+}\left(X_{i}\right)=\sum_{\substack{Y_{j} \cap X_{i} \neq \emptyset \\
\vee Y_{j}=\emptyset}} m_{X}\left(Y_{j}\right) \quad \mathrm{Pl}_{X}^{-}\left(X_{i}\right)=\sum_{Y_{j} \cap X_{i} \neq \emptyset} m_{X}\left(Y_{j}\right)
\end{aligned}
$$

Where $\mathrm{m}_{X}(S)$ is simply a function that returns the basic mass assigned to the set $S$ in the assignment $X$.
Together the two belief and two plausibility functions make pairings. $\mathrm{Bel}^{+}()$and $\mathrm{Pl}^{+}()$both consider inconsistency, $\mathrm{Bel}^{-}()$and $\mathrm{Pl}^{-}()$do not.

\section{Context}

Assignment to inconsistency corresponds to an assignment of mass to $\emptyset$ where both possibilities, $T$ and $\perp$, have been eliminated. The logic we will pursue restricts possibilities and so moves from $\{\top, \perp\}$, representing the possibility of both true and false i.e. ignorance, through to $\emptyset$ representing over restriction or inconsistent. Mass assignment is an extension and rationalisation of Dempster/Shafer theory of evidence (8) and (9) in that both use random sets and assign mass to those sets. Belnap (6) addresses the problems of reasoning with inconsistency but uses an evidential system that increases evidence from ignorance, where nothing is known, to having positive evidence whereas Baldwin (5) and this paper restrict possible values from everything is possible down to restricted values where all possibilities have been eliminated. In the context of this paper Belnap introduces inconsistency into his system by means of a setup, Baldwin takes evidence from the world and introduces it into the reasoning system by means of a semantic unification operator. Semantic unification is basically a specialised conditional probability operator based on probability intervals which allows claims to be made about the world conditional upon the evidence of the world supporting the claim, Dekhtyar et al. (4) address a similar problem on conditionalisation for interval probabilities but do not address the treatment of inconsistency. The complementary operator as addressed in this paper, semantic separation, is a form of abduction which takes the claims calculated by the reasoning system and applies them to real world values. In this paper we are primarily concerned with an inconsistent world and the impact it has on these two operators and the structure of the claims that a reasoning system may make about the world. Dekhtyar et al. (4) propose a definition of consistency which is shown in Definitions 1 and 2.

Definition 1: Let $\mathrm{V}$ be a set of random variables and $P$ : $\operatorname{dom}(V) \rightarrow 2^{[0,1]}$ be an interval probability distribution over V. A probabilistic interpretation $I_{V}$ satisfies $P\left(I_{V}=P\right)$ iff $(\forall \overline{(x)} \in \operatorname{dom}(V))\left(l_{\bar{x}} \leq I_{V}(\bar{x}) \leq u_{\bar{x}}\right)$.

Definition 2: An interval probability distribution $P$ : $\operatorname{dom}(V) \rightarrow 2^{[0,1]}$ is consistent iff there exists a pinterpretation $I_{V}$, such that $I_{V}=P$.

Dekhtyar et al. follow this up with Theorem 1

Theorem 1: Let $\mathrm{V}$ be a set of random variables and $P$ : $\operatorname{dom}(V) \rightarrow 2^{[0,1]}$ be an interval probability distribution over $\mathrm{V}$. Let $\operatorname{dom}(V)=\left\{\bar{x}_{1}, \ldots, \bar{x}_{m}\right\}$ and $P\left(\bar{x}_{i}\right)=\left[l_{i}, u_{i}\right]$. P is consistent iff the following conditions hold.

(i) $\sum_{i=1}^{m} l_{i} \leq 1$

(ii) $\sum_{i=1}^{i} u_{i} \geq 1$

The following table allows us to explore the definitions:

$m_{\{\}}=0.2 \quad m_{\{a\}}=0.1$

$m_{\{b\}}=0.0 \quad m_{\{c\}}=0.1$

$m_{\{a, b\}}=0.5 \quad m_{\{a, c\}}=0.0$

$m_{\{b, c\}}=0.1 \quad m_{\{a, b, c\}}=0.0$ 
This set of mass assignments is inconsistent as there is mass assigned to the empty set. They also give rise to a set of beliefs and plausibilities.
$\mathrm{Bel}^{+}(\{\})=0.2$
$\mathrm{Bel}^{+}(\{a\})=0.3$
$\mathrm{Bel}^{+}(\{b\})=0.2$
$\mathrm{Bel}^{+}(\{c\})=0.3$
$\mathrm{Pl}^{+}(\{\})=1.0$
$\mathrm{Pl}^{+}(\{a\})=0.8$
$\mathrm{Pl}^{+}(\{b\})=0.9$
$\mathrm{Pl}^{+}(\{c\})=0.4$
$\mathrm{Bel}^{+}(\{a, b\})=0.8$
$\mathrm{Pl}^{+}(\{a, b\})=0.9$
$\mathrm{Bel}^{+}(\{a, c\})=0.4$
$\mathrm{Pl}^{+}(\{a, c\})=1.0$
$\mathrm{Bel}^{+}(\{b, c\})=0.4 \quad \mathrm{Pl}^{+}(\{b, c\})=0.9$

Dekhtyar's conditions for consistency are as below:

(i) $\forall_{i=\{a\}}^{\{c\}} \mathrm{Bel}^{+}(i) \leq \mathrm{Pl}^{+}(i)$

(ii) $\sum_{i=\{a\}}^{\{c\}} \mathrm{Bel}^{+}(i)=0.8 \leq 1.0$

(iii) $\sum_{i=\{a\}}^{\{c\}} \mathrm{Pl}^{+}($i $)=2.1 \geq 1.0$

Clearly the equations above, and therefore the assignment, satisfy Dekhtyar's definition. The table is inconsistent as assignment to $\emptyset$ states that all possibilities have been eliminated and so Dekhtyar's definition does not go far enough. In dealing with probability intervals we need to consider the families of probabilities and reduce the assignments to the basic masses, from which beliefs and plausibilities may be derived. The mass assignment above gives a similar result for $\mathrm{Bel}^{-}()$and $\mathrm{Pl}^{-}()$.

\section{Semantic Unification and Separation}

\section{A. Semantic Unification}

Patching et al. (1) derive a version of semantic unification, shown in Equation 1 that deals with inconsistency.

$$
\begin{aligned}
& (X \mid Y)=R=\left\{R_{i j}: r_{i j}\right\} \\
& R_{i j}=\left\{\begin{array}{c}
\emptyset \text { if }\left(Y_{j}=\emptyset\right) \wedge\left(X_{i} \neq \emptyset\right) \\
\{\top\} \text { if }\left(Y_{j}=X_{i}=\emptyset\right) \\
\{\top\} \text { if }\left(Y_{j} \subseteq X_{i}\right) \wedge\left(X_{i} \neq \emptyset\right) \wedge\left(Y_{j} \neq \emptyset\right) \\
\{\perp\} \text { if }\left(X_{i} \cap Y_{j}=\emptyset\right) \wedge\left(X_{i} \neq \emptyset\right) \wedge\left(Y_{j} \neq \emptyset\right) \\
\{\top, \perp\} \text { if }\left(Y_{j} \cap X_{i} \neq \emptyset\right) \wedge\left(Y_{j} \not X_{i}\right) \\
\{\top, \perp\} \text { if }\left(X_{i}=\emptyset\right) \wedge\left(Y_{j} \neq \emptyset\right) \\
\quad r_{i j}=x_{i} y_{j}
\end{array}\right.
\end{aligned}
$$

In this definition, Equation 1, $X$ is the claim and $Y$ is the evidence which supports the claim.

\section{B. Claims}

A claim is a mass assignment that purports to state a truth about the world. Although claims may be true, the only way to discover if they are actually true is to evaluate the claim based upon some evidence.

We follow the path used by (1) and (2) by assuming in the first place that all claims are unitary mass assignments where all the mass is associated with a single focal element i.e.

$$
X=X_{i}: 1.0 \wedge\left(\forall X_{j} \in \mathbb{C} \mid X_{j} \neq X_{i}\right) X_{j}: 0.0
$$

In this paper we obtain valuations of claims following semantic unification as it then becomes clear whether the subsequent separation has behaved properly.

\section{Semantic Separation}

Hinde et al. (2) develop an operator, shown in Equation 2, that deals with inconsistency.

$$
\begin{aligned}
& (X \mid Y)=R=\left\{R_{j}: r_{j}\right\} \\
& Y_{i j}^{\prime}=\left\{\begin{aligned}
& X_{i} \text { if }\left(R_{j}=\{\top\}\right) \\
& \mathbb{C}-X_{i} \text { if }\left(R_{j}=\{\perp\}\right) \\
& \mathbb{C} \text { if }\left(R_{j}=\{\top, \perp\}\right) \\
& \emptyset \text { if }\left(R_{j}=\emptyset\right)
\end{aligned}\right. \\
& y_{i j}^{\prime}=x_{i} r_{j}
\end{aligned}
$$

In Equation $2 X$ is the claim, $(X \mid Y)$ is the support that the claim has given the consequence $Y^{\prime} . Y^{\prime}$ is the consequence.

\section{Discussion}

These operators, semantic unification and separation, both preserve inconsistency. The internal reasoning system therefore maintains and reasons with inconsistency. The next section, and the focus of this paper, considers the combination of the operators and places requirements on the operators that insist that the evidence is recoverable.

\section{Unification and Separation combined}

This section begins with considering the requirements that the pair of operators must satisfy regardless of the internal representation of inconsistency. The main requirements of this work are that the information that is entered into the reasoning system is recoverable, if that is required.

\section{A. Requirements}

In this section $Y$ is the token representing the evidence, $X$ and $I$ as the claims made by the reasoning system supported by the evidence $Y$, and $Y^{\prime}$ is the consequence that emerges from the reasoning system. We require that $Y$ and $Y^{\prime}$ are in some reasonable sense equal.

The assumptions we shall use in this paper rest on unitary claims $X$, and subsequently $I$, that are supported by the evidence $Y$. The set of claims covered by Assumption 1 represent the claims that are based on consistent evidence, whereas the claim based on Assumption 2 is that the evidence is inconsistent. Under these two assumptions we are able to state our requirements of the combined operator.

Assumption 1: $X$ is a unitary mass assignment $X=X_{1}$ : 1.0 and $X_{1} \neq \emptyset$

Under Assumption 1 the preservation of inconsistency is not necessarily required, and so the following set of requirements are made about the mass assigned to consistent aspects of the evidence. Aspects of the evidence that are not mentioned in the reasoning system have their mass assigned to ignorance. The mass assigned to inconsistency in the evidence $Y$ could be preserved in the consequence $Y^{\prime}$, or ignored. If there is no claim about inconsistency then it will be assigned to ignorance. If there is a claim about inconsistency in the reasoning system then the mass 
assigned to inconsistency in the consequence will be taken from ignorance.

Requirement 1.1: The belief measure, $\mathrm{Bel}^{-}()$, in the consequence $Y^{\prime}$ must be equal to the belief in the evidence $Y$ of the claim $X$ given the evidence $Y$. As $X$ consists of the set of subsets $X_{i}$ this is expressed as:

$$
\mathrm{Bel}_{Y^{\prime}}^{-}\left(X_{i}\right)=\mathrm{Be}_{Y}^{-}\left(X_{i}\right)
$$

Requirement 1.2: The plausibility measure $\mathrm{Pl}^{+}()$of the consequence $Y^{\prime}$ must be equal to the plausibility measure of any claim $X$ given the evidence $Y$ :

$$
\mathrm{Pl}_{Y^{\prime}}^{+}\left(X_{i}\right)=\underset{Y}{\mathrm{Pl}^{+}}\left(X_{i}\right)
$$

Evidence we are concerned about should be preserved into and out of the reasoning process, however if the reasoning process makes no reference to a piece of evidence then the mass assigned to that evidence should be assigned to ignorance. The consequences that are recovered from the reasoning system should also not have more belief, $\left(\mathrm{Bel}^{-}()\right)$, in them than the initial evidence, however their plausibility will be affected by the preservation of inconsistency, or otherwise. If the unification step takes no note of the inconsistency of the evidence then inconsistency will become ignorance; we are therefore concerned that $\mathrm{Pl}^{+} 0$ is preserved but not necessarily $\mathrm{Pl}^{-}()$. It is quite reasonable to be unconcerned that the world is inconsistent, but it is unreasonable for the mass assigned to inconsistency to be transferred anywhere but inconsistency or ignorance, they are both forms of ignorance. This gives rise to the Requirement 1.3:

Requirement 1.3: The assignment of mass to inconsistency plus the assignment of mass to ignorance in the consequence $Y^{\prime}$ must be greater than or equal to the assignment of mass to the assignment of mass to inconsistency plus the assignment of mass to ignorance in the evidence $Y$ :

$$
\mathrm{m}_{Y^{\prime}}(\emptyset)+\mathrm{m}_{Y^{\prime}}(\mathbb{C}) \geq \mathrm{m}_{Y}(\emptyset)+\mathrm{m}_{Y}(\mathbb{C})
$$

If we care about the preservation of inconsistency then there must be a requirement to that effect. If we make no assertions about how this inconsistency is transferred through the reasoning system, this follows:

Requirement 1.4: The belief measure $\mathrm{Bel}^{+}()$in the consequence $Y^{\prime}$ in inconsistency must be equal to the belief in the evidence $Y$ in inconsistency:

$$
\mathrm{Bel}_{Y^{\prime}}^{+}(\emptyset)=\mathrm{Bel}_{Y}^{+}(\emptyset)
$$

This last requirement is very similar to Requirement 1.1, we are concerned that all aspects of evidence are treated reasonably equally. These requirements are considerably simpler and less restrictive than the requirements cited in $(1 ; 2)$ but apply to the whole process instead of to each function separately. They do not constrain the reasoning system to include inconsistency, but it must preserve it for the consequences. The operators developed in (1) and (2) satisfy the new requirements, as should some others.

\section{B. Theorems}

In general, if $X=X_{1}: 1.0$ is an unitary claim, with all mass assigned to a single focal element then a general piece of evidence $(Y)$ consists of four main parts when compared to the generic claim $X$. They are:

1) The empty set $\emptyset$

2) Focal Sets $S=\left\{S_{p}\right\}$ where $\left(\forall S_{p} \in S\right)$ : $S_{p} \subseteq$ $X_{1} \wedge S_{p} \neq \emptyset$

3) Focal Sets $D=\left\{D_{q}\right\}$ where $\left(\forall D_{q} \in D\right)$ : $D_{q} \cap X_{1}=$ $\emptyset \wedge D_{q} \neq \emptyset$

4) Focal Sets $C=\left\{C_{r}\right\}$ where $\left(\forall C_{r} \in C\right)$ : $C_{r} \cap X_{1} \neq$ $\emptyset \wedge C_{r} \not X_{1}$

These four sets describe all possible focal elements of $Y$ with respect to how they relate to $X_{1} . S$ is all the focal elements that are subsets of $X_{1}$ (but are not the empty set, which is a special case), $D$ is all the disjoint focal elements with respect to $X_{1}$, and $C$ is all the focal elements conjoint with $X_{1}$ but not a subset.

$$
\begin{aligned}
Y= & \left\{\emptyset: \operatorname{m}_{Y}(\emptyset)\right\} \cup\left\{S_{p}: \operatorname{m}_{Y}\left(S_{p}\right)\right\} \cup \\
& \left\{D_{q}: \operatorname{m}_{Y}\left(D_{q}\right)\right\} \cup\left\{C_{r}: \operatorname{m}_{Y}\left(C_{r}\right)\right\}
\end{aligned}
$$

The consequence $\left(Y^{\prime}\right)$ consists of four main parts when compared to the generic claim $X$. They are:

1) The empty set $\emptyset$

2) Focal Sets $X_{i}$

3) Focal Sets $\mathbb{C}-X_{i}$

4) Focal Sets $\mathbb{C}$

These four sets describe all the focal elements of $Y^{\prime}$ and how they relate to $X_{i}$, making up the consequence $Y^{\prime}$.

We now address the relationship between $Y$ and $Y^{\prime}$ deriving $Y^{\prime}$ from $Y$ through $(X \mid Y)$, this is:

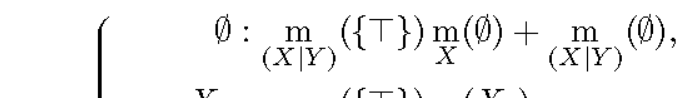

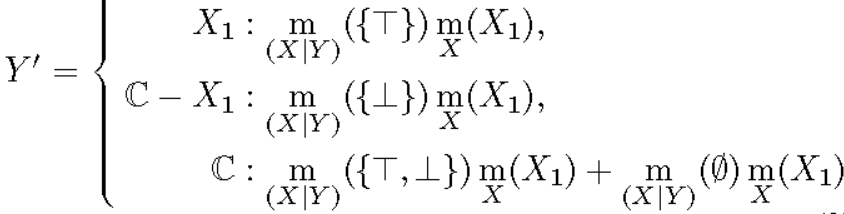

Substituting to express this in terms of $Y$ we get:

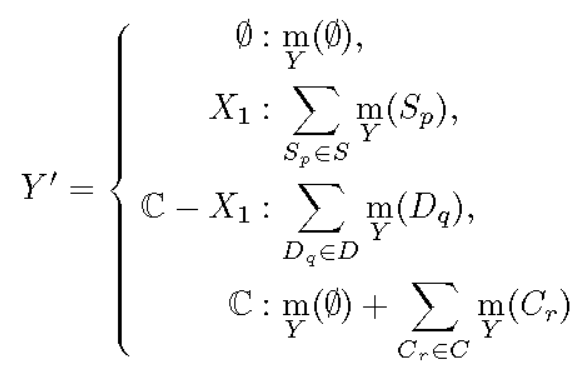

If we care about the preservation of inconsistency then we need Requirement 1.4. It is straightforward to check that the original unification and separation operators are still correct. 
Using the definition of semantic separation in Equation 2 on a unitary claim $X$, consisting of one element $X_{1}$ the separation of the general mass assignment claim $X$ and the general consequence $Y^{\prime}$ is given by Equation 3 .

Theorem 2: The combined functions (Equations 1) and (Equation 2) fulfil Requirement 1.1 (preservation of $\mathrm{Bel}^{-}$).

Proof: The belief in the general claim for the evidence has not changed:

$$
\underset{Y^{\prime}}{\mathrm{Bel}^{-}}\left(X_{1}\right)=\sum_{S_{p} \in S} \underset{Y}{m}\left(S_{p}\right)=\mathrm{Bel}_{Y}^{-}\left(X_{1}\right)
$$

The belief measures in $X_{1}\left(\mathrm{Bel}^{-}\right)$stay the same.

Theorem 3: The combined functions (Equations 1) and (Equation 2) fulfil Requirement 1.2 .

Proof:

$\mathrm{Pl}_{Y^{\prime}}^{+}\left(X_{1}\right)={\underset{Y}{Y}}_{(\emptyset)}\left(\emptyset \sum_{S_{p} \in S} \underset{Y}{\mathrm{~m}}\left(S_{p}\right)+\sum_{C_{r} \in C} \underset{Y}{\mathrm{~m}}\left(C_{r}\right)=\mathrm{Pl}_{Y}^{+}\left(X_{1}\right)\right.$

The plausibility measures $\left(\mathrm{Pl}^{+}\right)$stay the same.

Theorem 4: The combined functions (Equations 1) and (Equation 2) fulfil Requirement 1.3 .

Proof:

$\underset{Y^{\prime}}{\mathrm{Pl}^{+}}\left(X_{1}\right)=\operatorname{m}_{Y}(\emptyset)+\sum_{S_{p} \in S} \underset{Y}{m}\left(S_{p}\right)+\sum_{C_{r} \in C} \underset{Y}{m}\left(C_{r}\right)=\mathrm{Pl}_{Y}^{+}\left(X_{1}\right)$

The plausibility measures $\left(\mathrm{Pl}^{+}\right)$do not decrease.

Theorem 5: The combined functions (Equations 1) and (Equation 2) fulfil Requirement 1.4 .

Proof:

$$
\underset{\mathrm{Bel}^{\prime}}{\mathrm{Bel}^{+}}(\emptyset)=\underset{X}{m}(\emptyset)=\underset{Y}{\mathrm{Bel}^{+}}(\emptyset)
$$

The belief measures in inconsistency $\left(\mathrm{Bel}^{+}\right)$stay the same.

\section{Reasoning about inconsistency}

Section V-A dealt with the operators developed and refined in (1) and (2) which dealt with inconsistency by carrying it with the claim about the property we were interested in. It is perfectly reasonable that we may not be interested in the inconsistency and so we need an operator that can selectively ignore inconsistency. For this to be achieved the claims about properties need to have the inconsistency assigned to ignorance, as would a claim about another property, such as "greenness", when referring to "redness". We therefore need a claim about inconsistency, $I$.

Assumption 2: The unitary mass assignment $I$ represents the claim 'The evidence is inconsistent' $I=\emptyset: 1.0$

It is clear that introducing the claim $I$ in Assumption 2 does not affect the transfer of belief from $Y$ to $Y^{\prime}$ for the nonempty sets. It only remains to check that the mass assigned to inconsistency is correctly transferred through the claim $I$ :

$$
\begin{aligned}
\mathrm{m}_{(I \mid Y)}(\{\top\}) & =\mathrm{m}_{Y}(\emptyset), \\
\operatorname{m}_{(I \mid Y)}(\{\top, \perp\}) & =1.0-\mathrm{m}_{Y}(\emptyset), \\
\operatorname{Y}^{\prime}(\emptyset) & =\operatorname{m}_{(I \mid Y)}(\{\top\})=\mathrm{m}_{Y}(\emptyset)
\end{aligned}
$$

Mass assignments to $I$ contain no inconsistency but inconsistency is restored as it is transferred to $Y^{\prime}$.

There is no real need to transfer inconsistency into the reasoning system if it can be restored on exiting. We may need to represent the fact that inconsistency is a property of the evidence. Rather than propagate inconsistency through the reasoning we can reason about it just as any other property. The use of inconsistency in the unification operator may therefore be removed, but we still need to meet the requirements above. A definition of a suitable version of semantic unification is given in Equation 5. The only difference between this operator and the one given in Equation 1 is the topmost entry which changes from $\emptyset$ to $\{\top, \perp\}$.

$$
\begin{aligned}
& (X \mid Y)=R=\left\{R_{i j}: r_{i j}\right\} \\
& R_{i j}=\left\{\begin{aligned}
&\{\top, \perp\} \text { if }\left(Y_{j}=\emptyset\right) \wedge\left(X_{i} \neq \emptyset\right) \\
&\{\top\} \text { if }\left(Y_{j}=X_{i}=\emptyset\right) \\
&\{\top\} \text { if }\left(Y_{j} \subseteq X_{i}\right) \wedge\left(X_{i} \neq \emptyset\right) \wedge\left(Y_{j} \neq \emptyset\right) \\
&\{\perp\} \text { if }\left(X_{i} \cap Y_{j}=\emptyset\right) \wedge\left(X_{i} \neq \emptyset\right) \wedge\left(Y_{j} \neq \emptyset\right) \\
&\{\top, \perp\} \text { if }\left(Y_{j} \cap X_{i} \neq \emptyset\right) \wedge\left(Y_{j} \not \subset X_{i}\right) \\
&\{\top, \perp\} \text { if }\left(X_{i}=\emptyset\right) \wedge\left(Y_{j} \neq \emptyset\right) \\
& \quad r_{i j}=x_{i} y_{j}
\end{aligned}\right.
\end{aligned}
$$

Using the semantic unification operator defined in Equation 5 we transfer each property of the world into a claim about the world, including inconsistency. If we examine the operator as shown we see that in rows two and three the component of $X$ which represents inconsistency behaves exactly as any other component. So if the claim contains inconsistency then the operator will make use of it. Typically the claims we are using are unitary claims and so a further claim is required as defined in Assumption 2. The claim $X$ will assign any inconsistency in the evidence $Y$ to $\{\top, \perp\}$. $I$ will assign any inconsistency in the evidence $Y$ to $\{\top\}$.

An interesting by-product of this revised operator is that both claims $X$ and $I$ represent families of probabilities and it is now possible, and reasonable, to ask questions such as "what is the probability that the evidence is inconsistent?". By combining the claims and treating inconsistency as just another property we may obtain various probability estimates in the conventional way. Before we run away with the idea that there has been no change from the original semantic unification operators we note the original operators defined in the work on Fril, (3) did not allow statements involving inconsistency except to move all mass to unknown, $\{\top, \perp\}$.

\section{Resolution of inconsistency in the conclusions}

The conclusion based on the claim $X$ does not contain any inconsistency, however it must be resolved with the conclusion based on the claim $I$, which does contain inconsistency. The most general solution to this resolution is to take mass from the lower cardinality sets and not from ignorance; whereas the solution we want is to take the mass from ignorance and move it to inconsistency as we would with any other property of interest. Pragmatically, we would 
start with the consequence resulting from $I$ and then merging other quantities as they are extracted from the reasoning system by taking mass from ignorance.

Given that we employ the strategy advocated above to resolve the unitary statements and $\mathrm{m}_{X}\left(X_{1}\right)=1.0$ the following equations relate $Y$ to $Y^{\prime}$.

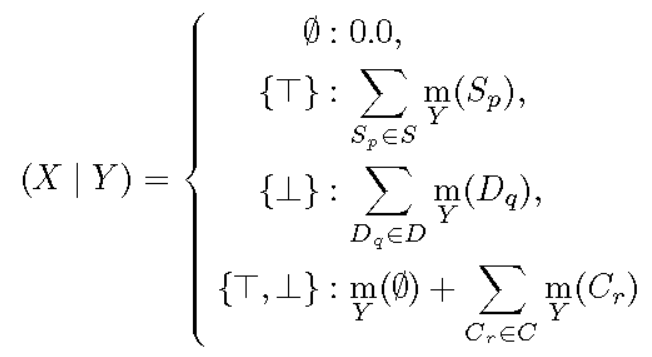

The claim $I$ consists of the single statement that the evidence is inconsistent and $\mathrm{m}_{I}(\emptyset)=1.0$.

$$
(I \mid Y)=\left\{\begin{array}{c}
\emptyset: 0.0, \\
\{\top\}:{\underset{Y}{Y}}_{(\emptyset),} \\
\{\perp\}: 0.0, \\
\{\top, \perp\}: 1.0-{\underset{Y}{Y}}(\emptyset)
\end{array}\right.
$$

So all $I$ does is measure the degree of inconsistency in the evidence $Y$.

The resultant $Y^{\prime}$ is then easily calculated as:

$$
Y^{\prime}=\left\{\begin{aligned}
\emptyset & : \operatorname{m}_{(I \mid Y)}(\{\top\})=m_{Y}(\emptyset), \\
X_{1} & : \sum_{S_{p} \in S} \mathrm{~m}_{Y}\left(S_{p}\right), \\
\mathbb{C}-X_{1} & : \sum_{D_{q} \in D} \mathrm{~m}_{Y}\left(D_{q}\right), \\
\mathbb{C} & : \sum_{C_{r} \in C} \mathrm{~m}_{Y}\left(C_{r}\right)
\end{aligned}\right.
$$

The mass assigned to $\mathbb{C}$ in $(X \mid Y)$ that is missing from the final assignment, $\mathrm{m}_{Y}(\emptyset)$, is precisely the amount assigned to $\{\top\}$ in $(I \mid Y)$, and is precisely the amount assigned to $\emptyset$ in the final assignment. It is also precisely the amount assigned to $\emptyset$ in the original evidence $Y$.

We are now in a position to formally prove the revised operators fulfil the requirements.

Theorem 6: The combined functions (Equations 5) and (Equation 2) fulfil Requirement 1.1 (preservation of $\mathrm{Bel}^{-}$).

Proof:

$$
\underset{Y^{\prime}}{\mathrm{Bel}^{-}}\left(X_{1}\right)=\sum_{S_{p} \in S} \underset{Y}{m}\left(S_{p}\right)=\operatorname{Bel}_{Y}^{-}\left(X_{1}\right)
$$

The belief measures in $X_{1}\left(\mathrm{Bel}^{-}\right)$stay the same.

Theorem 7: The combined functions (Equations 5) and (Equation 2) fulfil Requirement 1.2 .

Proof:

$$
\underset{Y^{\prime}}{\mathrm{Pl}^{+}}\left(X_{1}\right)=\underset{Y}{\mathrm{~m}}(\emptyset)+\sum_{S_{p} \in S} \underset{Y}{\mathrm{~m}}\left(S_{p}\right)+\sum_{C_{r} \in C} \underset{Y}{\mathrm{~m}}\left(C_{r}\right)=\mathrm{Pl}_{Y}^{+}\left(X_{1}\right)
$$

The plausibility measures $\left(\mathrm{Pl}^{+}\right)$stay the same.

Theorem 8: The combined functions (Equations 5) and (Equation 2) fulfil Requirement 1.3 .

Proof:

$$
\underset{Y^{\prime}}{\mathrm{Pl}^{+}}\left(X_{1}\right)=\underset{Y}{\mathrm{~m}}(\emptyset)+\sum_{S_{p} \in S} \underset{Y}{m}\left(S_{p}\right)+\sum_{C_{r} \in C} \underset{Y}{\mathrm{~m}}\left(C_{r}\right)=\mathrm{P}_{Y}^{+}\left(X_{1}\right)
$$

The plausibility measures $\left(\mathrm{Pl}^{+}\right)$do not decrease.

Theorem 9: The combined functions (Equations 5) and (Equation 2) fulfil Requirement 1.4 .

Proof:

$$
\mathrm{Bel}_{Y^{\prime}}^{+}(\emptyset)=\underset{I}{m}(\{\top\})=\mathrm{Bel}_{Y}^{+}(\emptyset)
$$

The belief measures in inconsistency $\left(\mathrm{Bel}^{+}\right)$stay the same.

\section{E. Eliminating inconsistency in the conclusion}

So far we have concerned ourselves with the restoration of inconsistency to the outside world. This may not be what is required. The restoration of inconsistency was achieved by mapping a claim $I^{\prime}$ in the conclusions to the claim $(I \mid Y)$ within the reasoning system to restore inconsistency. All that claim did was to take the token representing inconsistency within the claim and restore inconsistency. We could just as easily have taken a token $\omega$ and used it just as we used $X_{i}$ to form our conclusion. The conclusion would then have two components $\omega$ and $X_{i}$, where $X_{i}$ represents the consistent components of the statement.

\section{F. Using a token $\omega$ to represent inconsistency}

Let the final set of claims be as above but with $J^{\prime}=\{\omega\}$ : 1.0 replacing $I^{\prime}=\emptyset: 1.0$.

This is superficially reasonable and in many cases it may well be adequate. However, if we examine the possible movements of mass it becomes clear that although mass may be moved downwards towards inconsistency, it cannot be moved downwards towards $\{\omega\}$. It is also clear that care must be taken in the internal reasoning process as mass that would have been assigned to $\emptyset$ is now assigned to $\{\top, \perp\}$ thus allowing it to be moved downwards to other sets. The reasoning system must be aware of the representation of inconsistency. The property of inconsistency mentioned above may be easily replicated by including $\omega$ in all sets, including $\mathbb{C}$. The unification operator which achieves this is given in Equation 7.

$$
\begin{aligned}
& (X \mid Y)=R=\left\{R_{i j}: r_{i j}\right\} \\
& R_{i j}=\left\{\begin{array}{c}
\{\omega\} \text { if }\left(Y_{j}=\emptyset\right) \wedge\left(X_{i} \neq \emptyset\right) \\
\{\omega, \top\} \text { if }\left(Y_{j}=X_{i}=\emptyset\right) \\
\{\omega, \top\} \text { if }\left(Y_{j} \subseteq X_{i}\right) \wedge\left(X_{i} \neq \emptyset\right) \wedge\left(Y_{j} \neq \emptyset\right) \\
\{\omega, \top\} \text { if }\left(X_{i} \cap Y_{j}=\emptyset\right) \wedge\left(X_{i} \neq \emptyset\right) \wedge\left(Y_{j} \neq \emptyset\right) \\
\{\omega, \perp\} \text { if }\left(Y_{j} \cap X_{i} \neq \emptyset\right) \wedge\left(Y_{j} \not X_{i}\right) \\
\{\omega, \top, \perp\} \text { if }\left(X_{i}=\emptyset\right) \wedge\left(Y_{j} \neq \emptyset\right) \\
\quad r_{i j}=x_{i} y_{j}
\end{array}\right.
\end{aligned}
$$


The corresponding semantic separation operator is given in Equation 8.

$$
\begin{gathered}
(X \mid Y)=R=\left\{R_{j}: r_{j}\right\} \\
Y_{i j}^{\prime}=\left\{\begin{aligned}
& X_{i} \text { if }\left(R_{j}=\{\omega, \top\}\right) \vee\left(R_{j}=\{\top\}\right) \\
& \mathbb{C}-X_{i} \text { if }\left(R_{j}=\{\omega, \perp\}\right) \vee\left(R_{j}=\{\perp\}\right) \\
& \mathbb{C} \text { if }\left(R_{j}=\{\omega, \top, \perp\}\right) \vee\left(R_{j}=\{\top, \perp\}\right) \\
& \emptyset \text { if }\left(R_{j}=\{\omega\}\right) \vee\left(R_{j}=\emptyset\right) \\
& y_{i j}^{\prime}=x_{i} r_{j}
\end{aligned}\right.
\end{gathered}
$$

The semantic separation operator shown in Equation 8 is suitable for a reasoning system that preserves inconsistency, represents it as a single unitary claim or represents it using a token such as the one adopted in this paper, $\omega$. Clearly the transfer of inconsistency into the reasoning system must be performed by different semantic unification operators, whereas separation can be performed by a single operator suitably configured. To be consistent with the treatment of the other operators we need to prove that the transfer is correct.

Theorem 10: The combined functions (Equations 7) and (Equation 8) fulfil Requirement 1.1 (preservation of $\mathrm{Bel}^{-}$).

Proof: The belief in the general claim for the evidence has not changed:

$$
\underset{Y^{\prime}}{\mathrm{Bel}^{-}}\left(X_{1}\right)=\sum_{S_{p} \in S} \underset{Y}{m}\left(S_{p}\right)=\operatorname{Bel}_{Y}^{-}\left(X_{1}\right)
$$

The belief measures in $X_{1}\left(\mathrm{Bel}^{-}\right)$stay the same.

Theorem 11: The combined functions (Equations 7) and (Equation 8) fulfil Requirement 1.2 .

\section{Proof:}

$\underset{Y^{\prime}}{\mathrm{Pl}^{+}}\left(X_{1}\right)=\mathrm{m}_{Y}(\emptyset)+\sum_{S_{p} \in S} \operatorname{m}_{Y}\left(S_{p}\right)+\sum_{C_{r} \in C} \underset{Y}{\operatorname{m}}\left(C_{r}\right)=\underset{Y}{\mathrm{Pl}} \mathrm{l}^{+}\left(X_{1}\right)$

The plausibility measures $\left(\mathrm{Pl}^{+}\right)$stay the same.

Theorem 12: The combined functions (Equations 7) and (Equation 8) fulfil Requirement 1.3 .

Proof:

$\mathrm{Pl}_{Y^{\prime}}^{+}\left(X_{1}\right)=\operatorname{m}_{Y}(\emptyset)+\sum_{S_{p} \in S} \mathrm{~m}_{Y}\left(S_{p}\right)+\sum_{C_{r} \in C} \underset{Y}{\mathrm{~m}}\left(C_{r}\right)=\mathrm{Pl}_{Y}^{+}\left(X_{1}\right)$

The plausibility measures $\left(\mathrm{Pl}^{+}\right)$do not decrease.

Theorem 13: The combined functions (Equations 7) and (Equation 8) fulfil Requirement 1.4 .

Proof:

$$
\mathrm{Bel}_{Y^{\prime}}^{+}(\emptyset)=\underset{X_{1}}{m}(\{\omega\})=\mathrm{Be}_{Y}^{+}(\emptyset)
$$

The belief measures in inconsistency $\left(\mathrm{Bel}^{+}\right)$stay the same.

The use of $\omega$ or some other suitable symbol to represent inconsistency allows us to use most of the usual operators in our reasoning system except they need to take account of $\omega$.

\section{Conclusions}

We have taken the semantic unification operator together with the separation operator and extended the possible internal representations so that the reasoning system may reason about inconsistency rather than reason with inconsistency. The reasoning system does not need to represent the inconsistency as inconsistency, merely a claim about inconsistency. A final operator designed to represent inconsistency within the claims used the symbol $\omega$ to be able to reason about inconsistency and to preserve some of the properties of inconsistency in that $\omega$ is a component of all the possibility sets.

The paper shows that if evidence can be entered into and extracted from a representation then that evidence must be represented correctly within the system. By only insisting that evidence must be recoverable we have shown that a variety of approaches may be taken, resulting in several unification operators. Because the representation will already have been chosen by the time extraction is performed the semantic separation operator can be implemented as a single operator that does not require modification depending on the representation used.

\section{References}

[1] R. Patching, C. Hinde, and S. McCoy, "Inconsistency and semantic unification," Fuzzy Sets and Systems, vol. 157, pp. 2513-2539, 2006.

[2] C. Hinde, R. Patching, and S. McCoy, "Inconsistency and semantic separation," in Proceedings of the 2004 UK Workshop on Computational Intelligence, M. Withall and C. Hinde, Eds., 2004, pp. 265-272.

[3] J. Baldwin and S. Zhou, "A fuzzy relational inference language," Fuzzy sets and Systems, vol. 24, pp. 1-26, 1984.

[4] A. Dekhtyar and J. Goldsmith, "Conditionalization for interval probabilities," in Proceedings of Workshop on Conditionals, Information, and Inference'02, LNAI, Berlin, 2003. Springer. Springer, Berlin, 2003. [Online]. Available: citeseer.ist.psu.edu/dekhtyar02conditionalization.html

[5] J. Baldwin, "A theory of mass assignments for artificial intelligence," in Fuzzy Logic and Fuzzy Control, IJCAI'91 Workshops on fuzzy logic and fuzzy control. Sydney, Australia: Springer-Verlag, Jan 1991, pp. 22-34.

[6] A. Belnap, "A useful four-valued logic," in Modern Uses of Multiple-Valued Logic, G. Epstein and J. Dunn, Eds. Boston: Reidel Publishing Company, 1977, pp. 7-37.

[7] P. Smets and R. Kennes, "The transferable belief model," Artificial Intelligence, vol. 66, pp. 191-234, 1994.

[8] A. P. Dempster, "Upper and lower probability inferences for families of hypothesis with monotone density ratios." Ann. Math. Statistics, vol. 40, pp. 953-969, 1969.

[9] G. Shafer, A Mathematical Theory of Evidence. Princeton Univ. Press. Princeton, NJ, 1976. 